\title{
Thermal Performance of Ground Heat Exchanger based on Infinite-length Linear Heat-source Model
}

\author{
Chao Jiang ${ }^{1, a}$, Yanling Guan ${ }^{1, b}$, Juanling Shi ${ }^{2, c}$ \\ ${ }^{1}$ The College of Environmental Science and Engineering, Chang'an University, Xi'an, China,710054 \\ ${ }^{2}$ Xi'an District Heating Company, Xi'an, China,710043 \\ a15091336209@126.com, bguanyl1@163.com, cwjrl2005@126.com
}

Keywords: ground source heat pump; infinite-length linear heat source model; long-term performance

\begin{abstract}
Based on the principle of superposition, the Infinite-length Linear Heat-source Model (ILM) is applied to the analysis of the long-term performance of Ground Heat Exchanger for Ground Source Heat Pump. The existing heat transfer models, including the Infinite-length Linear Heat-source Model (ILM), the Finite-length Linear Heat-source Model (FLM) and the Infinite-length Cylindrical Heat-source Model (ICM), are compared. It is found that for deep buried pipe, the results of the ILM are exactly the same with that of the FLM, while the results of ICM show large deviation. Comparing with FLM, ILM is of much higher calculation speed which may save lots of computer burden when applying to actual projects.
\end{abstract}

\section{Introduction}

A lot of researches on the heat transfer model of ground source heat pump were reported in literature home and abroad which can be divided into analytic and numerical methods [1-9]. The underground heat exchange of ground source heat pump is a long-term non-steady-state process. Unless long-term (at least one year) dynamic simulation has carried out, the ground heat exchanger performance analysis can not be predicted. As the temperatures of inlet and outlet water of heat pump vary with the underground temperature change, the traditional method of energy analysis can not be used to predict annual energy consumption. So, dynamic energy simulation software must be coupled with the process of ground heat exchanger to carry on energy analysis.

The results of numerical model which takes into account complex boundary conditions are not general, while that of analytical model whose boundary conditions are simple are common but accuracy is low. However, for long-term progress (month, year or even longer), the accuracy of analytical solutions meets project needs. In actual project the dimensionless criterion $F o=\frac{a t}{r^{2}}>20$ is used as criterion for small error $[1,10]$.

On the basis of the principle of superposition, the existing analytical models are used to analyze the ground heat exchanger performance under variable load and compared. The results show that the Infinite-length Linear Heat-source Model has a lot of advantages when used to analyze the heat transfer performance such as simple formula, higher calculation speed which is worth using in the design and analysis of ground heat exchanger system.

\section{Heat transfer model of underground exchanger}

There are mainly three heat transfer models of underground exchanger which are the Infinite-length Linear Heat-source Model (ILM), the Finite-length Linear Heat-source Model (FLM) and the Infinite-length Cylindrical Heat-source Model (ICM).

2.1. ILM

The soil is assumed to be infinite medium with uniform temperature distribution. The linear heat-source is turned on at zero time. The underground temperature distribution is [10, 11]: 


$$
T(r, \tau)-T_{0}=\frac{q_{l}}{2 \pi \lambda_{s}} \int_{X}^{\infty} \frac{e^{-\beta^{2}}}{\beta} d \beta=\frac{q_{l}}{2 \pi \lambda_{s}} \operatorname{Ei}(X) .
$$

Where

$$
X=\frac{r}{2 \sqrt{a \tau}} \text {; }
$$

$\mathrm{T}$ - the underground temperature with a distance of $\mathrm{r}$ to the linear heat source, ${ }^{\circ} \mathrm{C}$;

$T_{0}$ - the undisturbed temperature of soil, ${ }^{\circ} \mathrm{C}$;

$q_{1}$-linear heat source heat dissipation, $\mathrm{W} / \mathrm{m}$;

$r$-the distance to linear heat source, $\mathrm{m}$;

$\lambda_{\mathrm{s}}$ - thermal conductivity of soil, $\mathrm{W} /(\mathrm{m} \cdot \mathrm{K})$;

$a$-thermal diffusivity of soil, $\mathrm{a}=\lambda_{\mathrm{s}} / \rho_{\mathrm{s}} / c_{\mathrm{s}}$;

$\rho_{\mathrm{s}}$ - the density of soil, $\mathrm{kg} / \mathrm{m}^{3}$;

$C_{\mathrm{s}}$ — the specific heat of soil, $\mathrm{J} / \mathrm{kg} /{ }^{\circ} \mathrm{C}$;

$\tau$-the time from the start time, s.

\section{2. $I C M$}

Kavanaugh calculated the heat transfer and underground temperature using ICM. Assuming a separate column is surrounded by infinite homogeneous medium and the heat transfer process is purely heat conduction. The analytical solution model is as follow [12]:

$$
\theta(r, \tau)=\frac{q_{l}}{\pi^{2} \lambda_{s}} \int_{0}^{\infty}\left(e^{-u^{2} \tau}-1\right) \frac{J_{0}(u r) Y_{1}\left(u r_{b}\right)-Y_{0}\left(u r_{b}\right) J_{1}\left(u r_{b}\right)}{u^{2}\left[J_{1}^{2}\left(u r_{b}\right)+y_{1}^{2}\left(u r_{b}\right)\right]} d u .
$$

Where

$\theta$-dimensionless temperature, $\theta(r, \tau)=T(r, \tau)-T_{0}$;

$\boldsymbol{J}_{\mathbf{0}}, \boldsymbol{J}_{\mathbf{1}}, \boldsymbol{Y}_{\mathbf{0}}, \boldsymbol{Y}_{\mathbf{1}}$-Bessel functions of the first kind and Bessel functions of the second kind.

The analytical solution obtained by ICM which contains infinite integral of Bessel functions is very difficult to use. It is recommended to use the following approximate formula for calculation:

$$
\theta(r, \tau)=\frac{q_{l}}{2 k \pi r_{0}} \sum \frac{V_{j}}{j} \frac{K_{0}\left(\omega_{j} r\right)}{\omega_{j} K_{1}\left(\omega_{j} r_{0}\right)}
$$

Where

$$
\begin{aligned}
& \omega_{j}=\sqrt{\frac{j \ln 2}{a \tau}} \\
& V_{j}=\sum_{k=\operatorname{int}\left(\frac{j-1}{2}\right)}^{\min (j, 5)} \frac{(-1)^{j-5} k^{5}(2 k) !}{(5-k) !(k-1) ! k !(j-k) !(2 k-j) !}
\end{aligned}
$$

\subsection{FLM}

The borehole where the U-tube heat exchanger is laid which transfers heat with the soil is considered to be a finite linear heat source which exchanges heat with the semi-infinite medium. Green function and linear superposition principle are used. The analytical solution is obtained as follow [4]:

$$
\theta(\rho, z, \tau)=\frac{q_{l}}{4 \pi \lambda_{s}} \int_{0}^{H}\left\{\frac{\operatorname{erfc}\left[\frac{\sqrt{\rho^{2}+(z-h)^{2}}}{2 \sqrt{a \tau}}\right]}{\sqrt{\rho^{2}+(z-h)^{2}}}-\frac{\operatorname{erfc}\left[\frac{\sqrt{\rho^{2}+(z+h)^{2}}}{2 \sqrt{a \tau}}\right]}{\sqrt{\rho^{2}+(z+h)^{2}}}\right\} d h .
$$

Where 
$H$ - the depth of the hole, m;

$\rho$ - the radial distance to the linear heat source, $\mathrm{m}$;

$z$ - the distance to the ground, $\mathrm{m}$;

erfc - the complementary error function.

\subsection{The analytical model under variable load}

The above heat transfer models are proposed for the constant load, which can not be used in practical projects unless modified because the ground heat exchanger load varies with building load and COP of heat pump. The heat transfer equation for the constant-property medium is linear equation. Assuming that the ground heat exchanger load is constant in a small period of time (usually $1 \mathrm{~h}$ ), the underground temperature distribution can be analyzed by the principle of superposition using above three models $[13,14]$.

Assuming that the ground heat exchanger load is constant in the period of $\left(\tau_{\mathrm{i}-1}, \tau_{\mathrm{i}}\right)$, the underground temperature can be described by the following formula:

$$
\theta\left(r, \tau_{n}\right)=\sum_{i=1}^{n}\left(q_{i}-q_{i-1}\right) g\left(\tau_{n}-\tau_{i-1}\right)+q_{n} R_{b}
$$

Where

$q_{\mathrm{i}}$ - the load of i time, $\mathrm{W} / \mathrm{m}$;

$\tau_{\mathrm{i}}$-the i time, s;

$\tau_{\mathrm{n}}$ - the end time, $\mathrm{s}$;

$g(\tau)$ - the temperature response coefficient;

$R_{b}$ - the borehole thermal resistance

For the pipe group which is composed by n U-tube, formula (4) is changed as follow on the base of the superposition principle:

$$
\theta\left(r, \tau_{m}\right)=\sum_{j=1}^{n-1} \sum_{i=1}^{m}\left(q_{i}-q_{i-1}\right) g\left(\tau_{m}-\tau_{i-1}\right)+q_{m} R_{b} .
$$

Considering the borehole thermal resistance, the U-tube loop average temperature can be obtained by:

$$
T_{f}(\tau)=T\left(r_{b}, \tau\right)+q_{l}(\tau) R_{b} .
$$

Where

$r_{b}$-Borehole radius, $\mathrm{m}$.

For each discrete time, Eq. 6 changes as follows:

$$
\begin{aligned}
& T_{f}\left(\tau_{i}\right)=T_{0}+\sum_{j=1}^{i}\left(q_{j}-q_{j-1}\right) g\left(\tau_{i}-\tau_{j-1}\right)+q_{i} R_{b} . \\
& T_{f, o}\left(\tau_{i}\right)=2 T_{f}\left(\tau_{i}\right)-\frac{q}{c m} . \\
& T_{f, i}\left(\tau_{i}\right)=2 T_{f}\left(\tau_{i}\right)-T_{f, o}\left(\tau_{i}\right) .
\end{aligned}
$$

Where

$T_{f, o}$ - the outlet temperature of U-tube, ${ }^{\circ} \mathrm{C}$;

$T_{f, i}$ - the inlet temperature of U-tube, ${ }^{\circ} \mathrm{C}$;

${ }^{2}$ - the specific heat of the circulating fluid, $\mathrm{J} /(\mathrm{kg} \cdot \mathrm{K})$;

$\mathrm{m}$ - the mass flow of the circulating fluid, $\mathrm{kg} / \mathrm{s}$. 


\section{Comparison of the above three analytical model}

According to formula (4) it can be seen that the main difference of the above three analytical models for heat transfer analysis of ground heat exchanger is reflected in the different temperature response coefficient $g(\tau)$. Thus the comparison of the three heat transfer model can be carried out by the comparison of $g(\tau)$.

\subsection{The expression of $g(\tau)$}

For ILM, $g(\tau)$ can be written as:

$$
g(\tau)=\frac{1}{4 \pi \lambda_{s}} E i\left(\frac{r_{b}^{2}}{4 a \tau}\right)
$$

For ICM, it is:

$$
g(\tau)=\frac{1}{\pi^{2} \lambda_{s}} \int_{0}^{\infty}\left(e^{-u^{2} \tau}-1\right) \frac{J_{0}(u r) Y_{1}\left(u r_{b}\right)-Y_{0}\left(u r_{b}\right) J_{1}\left(u r_{b}\right)}{u^{2}\left[J_{1}^{2}\left(u r_{b}\right)+y_{1}^{2}\left(u r_{b}\right)\right]} d u .
$$

For FLM, it is:

$$
g(\tau)=\frac{1}{4 \pi \lambda_{s}} \int_{0}^{H}\left\{\frac{\operatorname{erfc}\left[\frac{\sqrt{\rho^{2}+(z-h)^{2}}}{2 \sqrt{a \tau}}\right]}{\sqrt{\rho^{2}+(z-h)^{2}}}-\frac{\operatorname{erfc}\left[\frac{\sqrt{\rho^{2}+(z+h)^{2}}}{2 \sqrt{a \tau}}\right]}{\sqrt{\rho^{2}+(z+h)^{2}}}\right\} d h .
$$

\subsection{Comparison}

The comparison of $g(\tau)$ for the three analytical models is shown as Fig. 1-3. The different curves of $g$ $(\tau)$ for one year $(8760 \mathrm{~h})$ of three models are drawn in the same coordinate plane.

The solutions of ILM and ICM are independent of depth while that of FLM not. It can be observed that $g(\tau)$ increases with time for each model. It has a faster increment at the beginning and trends toward constant. With the increase of the borehole depth, the results of ILM and FLM harmonize, while the result of ICM has a greater deviation with the former two.

For the common borehole with depth of $100 \mathrm{~m}$, the comparison of $\mathrm{g}(\tau)$ for ILM and FLM is shown as Figure 4. It can be found that, for the borehole with the depth of $100 \mathrm{~m}, I L M$ and FLM are exactly the same.

\subsection{Computing speed}

The computer which has been used to do the calculation is configured as Table 1 . To calculate $g(\tau)$ of 8760h for ILM it had taken 0.002278s, while FLM 7167.406341s. 


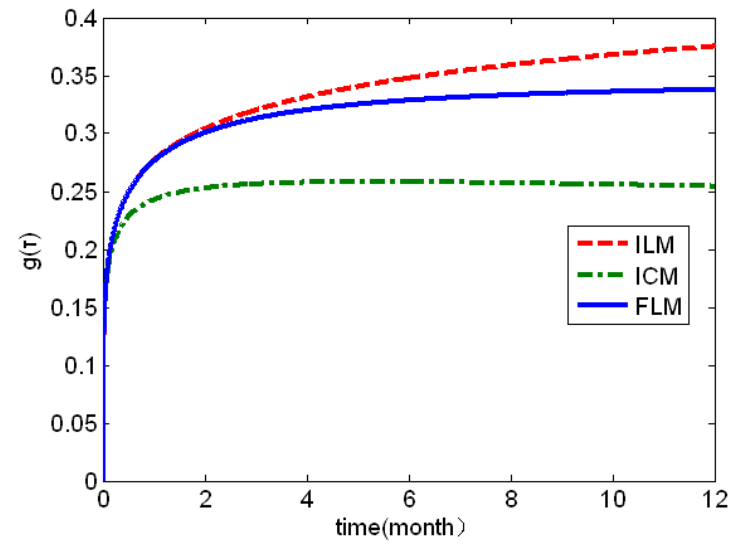

Fig. 1. Comparison of $\mathrm{g}(\tau)$ for $10 \mathrm{~m}$ depth borehole

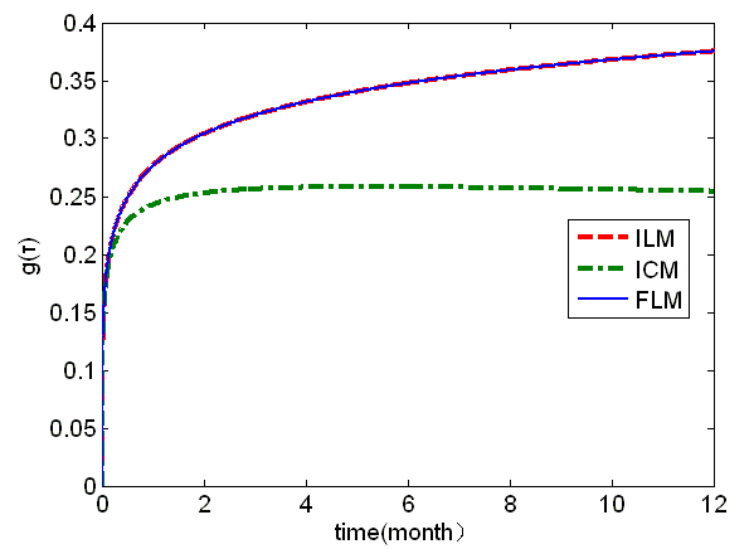

Fig. 3. Comparison of $g(\tau)$ for $30-160 m$ depth borehole

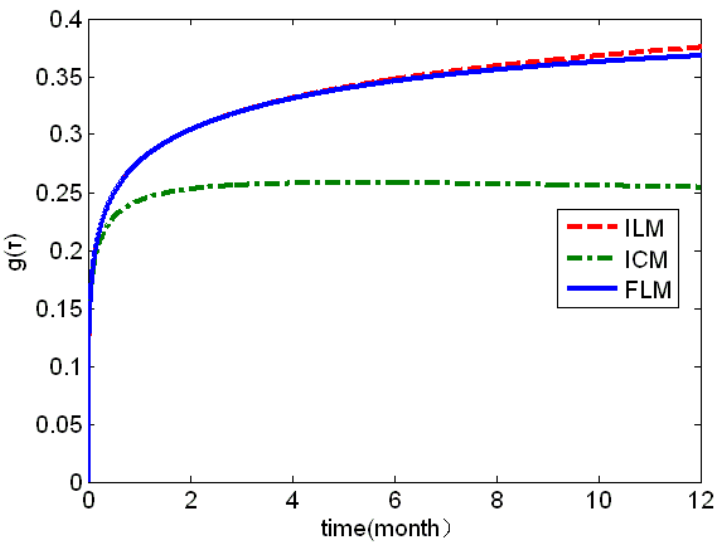

Fig. 2. Comparison of $\mathrm{g}(\tau)$ for $20 \mathrm{~m}$ depth borehole

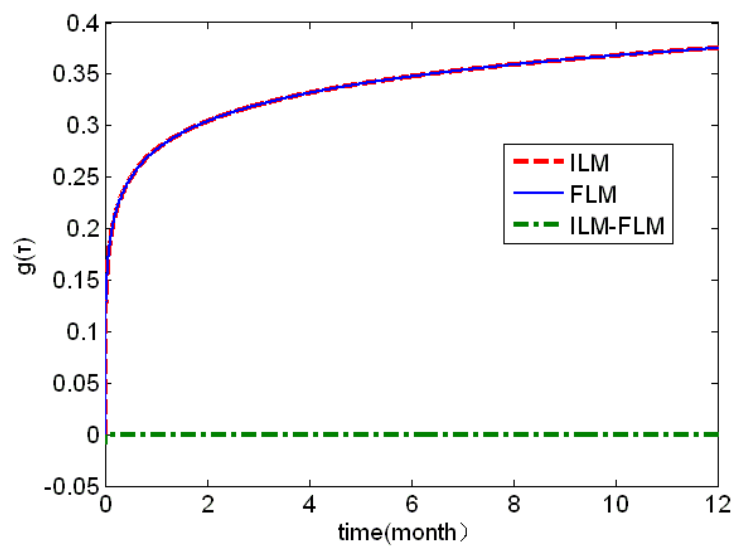

Fig. 4. Comparison of $g(\tau)$ for $100 \mathrm{~m}$ depth borehole of infinite-length linear heat source model and finite-length linear heat source model

Table 1. Computer configuration

\begin{tabular}{|l|l|}
\hline CPU & Intel(R) Core(TM)2 Duo CPU E4500 @ 2.20GHz \\
\hline BIOS & Award Software International, Inc.; F2 \\
\hline Motherboard & Gigabyte Technology Co., Ltd.;945PL-S3P \\
\hline Video card & NVIDIA GeForce 7300 GT \\
\hline Memory & DDR2 SDRAM;1024 MB;DDR2 667(PC5300) \\
\hline Hard disk & ST3160815AS;7200 rad/min;160.0 GB \\
\hline
\end{tabular}

To calculate $\mathrm{g}(\tau)$ for one year, the speed of ILM is 3.15 million times of FLM which suggests that ILM is excellent when using to analyze the thermal performance of the ground heat exchanger which is deep buried.

\section{Summary}

(1) For the ground source heat pump with deep buried pipes, the solution of ILM is the same as FLM, while ICM has larger deviations with them.

(2) For the annual simulation of $100 \mathrm{~m}$ depth borehole, the calculation speed of ILM is million times of FLM. 
(3) ILM can be applied to the analysis of the thermal performance for ground heat exchangers on the basis of the principle of superposition.

\section{Acknowledgement}

The research is supported by the Scientific and Technological Research and Development Program of Shaan xi (2013K13-02-01) and Xi'an District Heating Company.

\section{References}

[1] Ingersoll, L.R. and H.J. Plass. Theory of the Ground Pipe Heat Source for the Heat Pump. [J] Heating Piping \& Air Conditioning. 1948, 119-122.

[2] Muraya, N. K., Numerical Modeling of the transient thermal interference of vertical U-tube heat exchangers [D]. Ph.D. Thesis, Texas A\&M University, College Station, TX. 1995.

[3] Cenk Yavuzturk, Modeling of vertical ground loop heat exchangers for ground source heat pump systems [D] Ph.D. Thesis, the Faculty of the Graduate College of the Oklahoma State University. 1999.

[4] Zeng He yi, Diao Nai ren, Fang Zhao hong; A Model of Finite-length Linear Heat-source for the Vertical Embedded Pipe of a Ground-source Heat Pump [J] JOURNAL OF ENGINEERING FOR THERMAL ENERGY AND POWER, 2003, 18 (2) : 166-169

[5] Diao Nai ren, Zeng He yi, Fang Zhao hong; A Quasi Three-dimensional Heat Transfer Model for Vertical U-Tube Geothermal Heat Exchangers [J] JOURNAL OF ENGINEERING FOR THERMAL ENERGY AND POWER, 2003, 18 (4) : 387-390

[6] Tu Ai min, Dong Hua, Yang Wei bo, Tong Shao chen; SIMULATION RESEARCH OF VERTICAL U-TUBE GROUND HEAT EXCHANGERS BASED ON CYLINDRICAL HEAT SOURCE MODEL [J] ACTA ENERGIAE SOLARIS SINICA，2006，27（3）: 259-264

[7] Yang Wei bo, Shi Ming heng; A Study of Heat Exchange Characteristics of Vertical U-shaped Embedded Tubes Based on an Element Energy Balance Method[J] JOURNAL OF ENGINEERING FOR THERMAL ENERGY AND POWER, 2007, 22 (1) : 96-100

[8] Li Da peng, Liao Sheng ming: Three-dimensional numerical simulation and heat transfer analysis for U-tube ground heat exchangers [J]; HEATING VENTILATING \& AIR CONDITIONING, 2008, 38 (12)

[9] Chen Xiao chun, Xu Hai song, etc: Three-dimensional unsteady fluid-solid coupling heat transfer numerical simulation for U-tube ground heat exchangers [J] Construction Science and Technology, 2008, 10: 71-76

[10] Ingersoll, L. R., O. J. Zobel, and A. C. Ingersoll. Heat Conduction with Engineering, Geological, and Other Applications[M] New York: McGraw-Hill. 1954.

[11] Yavuzturk C. Modeling of vertical ground loop heat exchangers for ground source heat pump systems (Master's Degree Thesis)[D].Oklahoma (USA):Oklahoma State University,1995.

[12] Louis Lamarche A fast algorithm for the hourly simulations of ground-source heat pumps using arbitary response factors [J] Renewable Energy 34(2009) 2252-2258

[13] Heilstrǒm G, Ground heat storage, thermal analysis of duct storage systems. Sweden: Department of Mathematics Physics, University of Lund; 1991.

[14] Eskilson P. Thermal analysis of heat extraction boreholes. Sweden: Department of Mathematics Physics, University of Lund; 1987. 